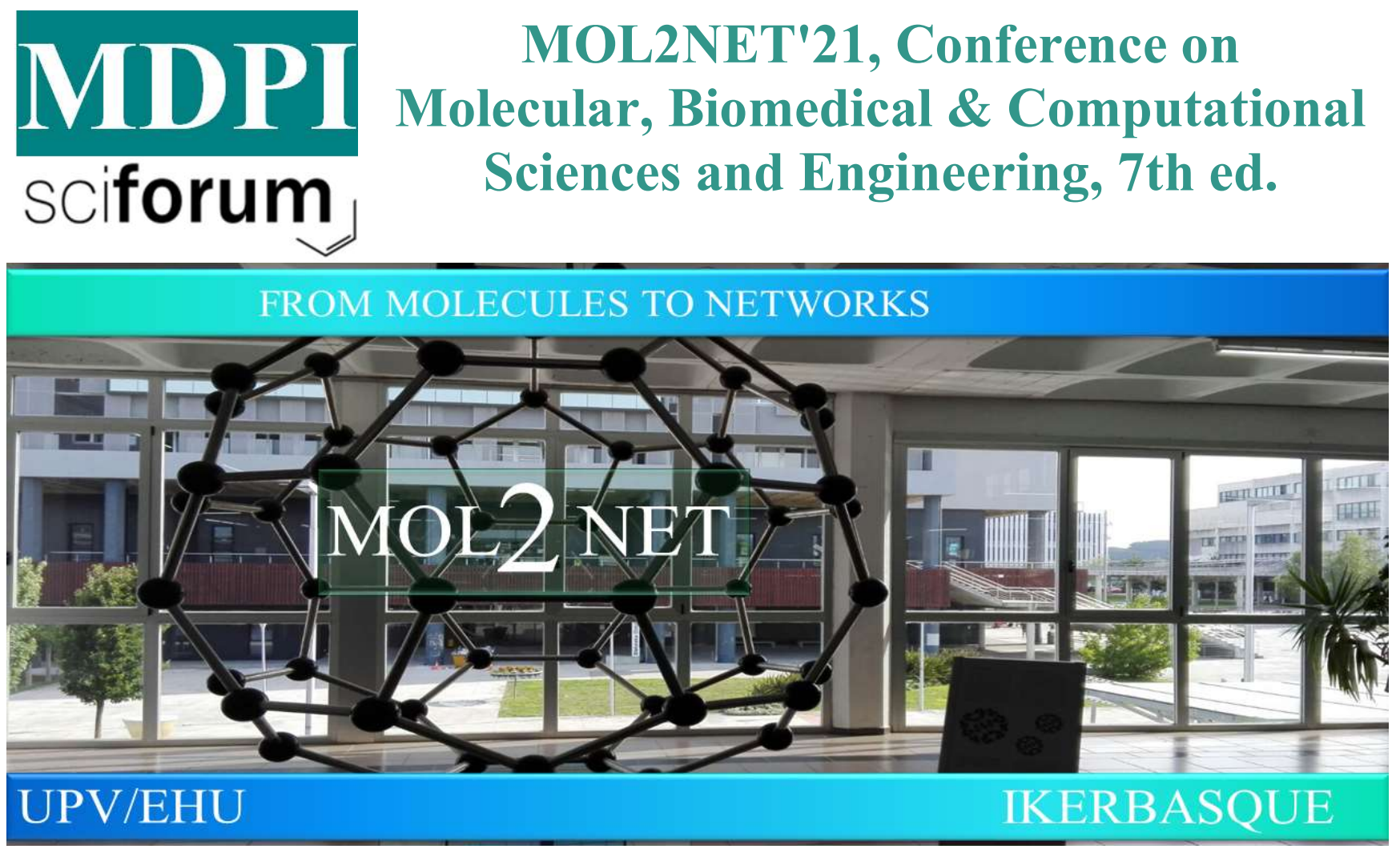

\title{
Silver nanoparticles via Passiflora incarnata: Synthesis, antioxidant and antimicrobial activity
}

Ioana-Raluca Suica-Bunghez (raluca_bunghez@yahoo.com) ${ }^{*}, 1$, Ana-Alexandra Sorescu (anaalexandra.sorescu@icechim.ro) ${ }^{*}$,.,Mariana Calin (mariana.calin@icechim.ro) ${ }^{1}$

${ }^{I}$ The National Research \& Development Institute for Chemistry and Petrochemistry

\begin{abstract}
Passiflora incarnata L. is a medicinal plant, used all over the world, especially for its contribution to reducing anxiety and stress. Passion flower compounds, leaves and flowers, presented beneficial properties for human body. Scientific studies have shown the antioxidant and antibacterial potential of flavonoids and tannins present in medicinal or ornamental plants. In this research paper, it is demonstrated the beneficial components of Passiflora incarnata L. leaves, quantitative (polyphenols, flavonoids, etc.) and qualitative (alkaloids, carbohidrates, terpenoids, etc) characterizing it. The next step was to developed a simple green and economical method for synthesis of silver nanoparticles (AgNPs) using this Passiflora incarnata L. plant. The analytical techniques studies suggested that Passiflora incarnata L. have played an important role in the reduction and stabilization of silver nanoparticles. Using UV-Vis method, it was observed an intense peak between 450-500 $\mathrm{nm}$ specific for silver nanoparticles. optical microscopy revealed the formation of spherical nanoparticles with size less than $100 \mathrm{~nm}$, after ultrasonication. The eco-friendly silver nanoparticles presented strong antioxidant properties, so AgNP-Passiflora incarnata L. leaves sample has a higher activity than mulberry extract sample. The antimicrobial activity of Passiflora incarnata L. was tested on Candida albicans yeast and it was observed an important antimicrobial activity.
\end{abstract}

